University of New Hampshire

University of New Hampshire Scholars' Repository

Space Science Center

Institute for the Study of Earth, Oceans, and

Space (EOS)

1997

\title{
The MeV spectrum of Cygnus X-1 as observed with COMPTEL
}

\author{
Mark L. McConnell \\ University of New Hampshire - Main Campus, mark.mcconnell@unh.edu \\ K Bennett \\ ESTEC \\ H Bloemen \\ Space Research Organization of the Netherlands \\ W Collmar \\ Max-Planck-Institut für extraterrestriche Physik \\ W Hermsen \\ Space Research Organization of the Netherlands (SRON)
}

See next page for additional authors

Follow this and additional works at: https://scholars.unh.edu/ssc

Part of the Astrophysics and Astronomy Commons

\section{Recommended Citation}

The MeV spectrum of Cygnus X-1 as observed with COMPTEL McConnell, M. and Bennett, $\mathrm{K}$. and Bloemen, H. and Collmar, W. and Hermsen, W. and Kuiper, L. and Much, R. and Ryan, J. and Schönfelder, V. and Steinle, H. and Strong, A. and van Dijk, R., AIP Conference Proceedings, 410, 829-833 (1997), DOl:http://dx.doi.org/10.1063/1.53984

This Conference Proceeding is brought to you for free and open access by the Institute for the Study of Earth, Oceans, and Space (EOS) at University of New Hampshire Scholars' Repository. It has been accepted for inclusion in Space Science Center by an authorized administrator of University of New Hampshire Scholars' Repository. For more information, please contact Scholarly.Communication@unh.edu. 


\section{Authors}

Mark L. McConnell, K Bennett, H Bloemen, W Collmar, W Hermsen, L Kuiper, R Much, James M. Ryan, V Schonfelder, H Steinle, A W. Strong, and R VanDijk 


\section{AIP $\mid$ proceedings}

\section{The MeV spectrum of Cygnus X-1 as observed with COMPTEL}

M. McConnell, K. Bennett, H. Bloemen, W. Collmar, W. Hermsen, L. Kuiper, R. Much, J. Ryan, V. Schönfelder, H. Steinle, A. Strong, and R. van Dijk

Citation: AIP Conference Proceedings 410, 829 (1997); doi: 10.1063/1.53984

View online: http://dx.doi.org/10.1063/1.53984

View Table of Contents:

http://scitation.aip.org/content/aip/proceeding/aipcp/410?ver=pdfcov

Published by the AIP Publishing

Articles you may be interested in

Gamma-ray spectral variability of Cygnus X-1

AIP Conf. Proc. 587, 96 (2001); 10.1063/1.1419379

The spectral variability of Cygnus $\mathrm{X}-1$ at $\mathrm{MeV}$ energies

AIP Conf. Proc. 510, 114 (2000); 10.1063/1.1303185

A thermal-nonthermal inverse Compton model for Cyg X-1

AIP Conf. Proc. 410, 868 (1997); 10.1063/1.54154

Modeling Cygnus X-1 y 2 spectra observed by BATSE AIP Conf. Proc. 410, 858 (1997); 10.1063/1.53989

RXTE observation of Cygnus X-1: spectra and timing AIP Conf. Proc. 410, 849 (1997); 10.1063/1.53987 


\title{
The MeV Spectrum of Cygnus X-1 as Observed with COMPTEL
}

\author{
M. McConnell*, K. Bennett ${ }^{\ddagger}$, H. Bloemen", W. Collmar , W. \\ Hermsen", L. Kuiper", R. Much ${ }^{\ddagger}$, J. Ryan*, V. Schönfelder", \\ H. Steinleף, A. Strongף, and R. van Dijk ${ }^{\ddagger}$
}

* University of New Hampshire, Durham, NH

${ }^{\ddagger}$ Astrophysics Division, ESTEC, Noordwijk, The Netherlands

\|SRON-Utrecht, Utrecht, The Netherlands

Max Planck Institute (MPE), Garching, Germany

\begin{abstract}
The COMPTEL experiment on the Compton Gamma-Ray Observatory (CGRO) has observed the Cygnus region on several occasions since launch. These data represent the most sensitive observations to date of Cygnus X-1 in the $0.75-30 \mathrm{MeV}$ range. The spectrum shows significant evidence for emission extending out to several $\mathrm{MeV}$. These data alone suggest a need to modify the thermal Comptonization models or to incorporate some type of non-thermal emission mechanism. Here we report on the results of an analysis of selected COMPTEL data collected during the first three years of the CGRO mission. These data are then compared with contemporaneous data from both BATSE-EBOP and OSSE. Given a lack of consistency between the OSSE and BATSE-EBOP spectra, it is difficult to draw firm conclusions regarding the exact shape of the spectrum near $1 \mathrm{MeV}$. A few general conclusions can, however, be drawn from these data.
\end{abstract}

\section{INTRODUCTION}

It has become increasingly apparent over the last several years that the standard thermal Comptonization model [1] does not provide an adequate description of the broad-band spectrum of Cyg X-1. Several modifications to the standard model have been proposed that seek to provide a better fit to the data. For example, modifications to the standard model have been developed which expand the range of allowable parameter space [2-4]. Other models have pursued alternative geometries that can also lead to improvements in the model. These include the incorporation of Compton backscatter radiation from a cooler optically-thick accretion disk $[5,6]$ or models based on a thermally stratified geometry $[7,8]$. Still other theorists have proposed schemes which are based on nonthermal acceleration processes [9]. All of these models have their 
merits. Unfortunately, given the quality of the available data, it is difficult to determine a clearly favored candidate to account for the observed spectrum near $1 \mathrm{MeV}$.

\section{OBSERVATIONS AND DATA ANALYSIS}

To date, COMPTEL has obtained numerous observations of the Cygnus region. Most of the high-quality (i.e., near on-axis) observations took place during the first three years of the mission. Here, we have selected a subset of these data for analysis (Table 1). The choice of observations was dictated by the availability of contemporaneous OSSE data. These data, along with contemporaneous results from BATSE, can, in principle, be used to assemble an improved picture of the spectrum near $1 \mathrm{MeV}$. In all cases except VP 318.1, the BATSE 45-140 keV flux level (as derived from Earth occultation analysis) is fairly constant at $\sim 0.1$ photons $\mathrm{cm}^{-2} \mathrm{sec}^{-1}$. During VP 318.1 , the hard $\mathrm{X}$-ray flux was lower by about a factor of five.

The analysis of COMPTEL data involves generating a series of images, one for each energy interval of interest. Assumptions regarding the spectral shape are incorporated into the point-spread-functions (PSFs) used in the analysis of each image. Flux values derived from each image are used to compile a spectrum of the source. The resulting spectrum is then compared versus that assumed for the PSF generation to insure a consistent analysis. The COMPTEL image analysis for Cyg X-1 is complicated by the fact that we are looking in the galactic plane. Images generated with COMPTEL data generally show some level of spatial structure, much of which is believed to result from galactic diffuse emission. In the present case, the spatial analysis of each energy

TABLE 1. List of COMPTEL observations used in the present analysis. The effective exposure is a measure of the equivalent on-axis exposure (measured in days), taking into account earth occultations, data gaps, etc.

\begin{tabular}{ccccccc}
\hline $\begin{array}{l}\text { Viewing } \\
\text { Period }\end{array}$ & $\begin{array}{c}\text { Start } \\
\text { Date }\end{array}$ & $\begin{array}{c}\text { Start } \\
\text { TJD }\end{array}$ & $\begin{array}{c}\text { End } \\
\text { Date }\end{array}$ & $\begin{array}{c}\text { End } \\
\text { TJD }\end{array}$ & $\begin{array}{c}\text { Viewing } \\
\text { Angle }\end{array}$ & $\begin{array}{c}\text { Effective } \\
\text { Exposure }\end{array}$ \\
\hline & & & & & & \\
2.0 & 30-May-1991 & 8406 & 8-Jun-1991 & 8415 & $1.7^{\circ}$ & 3.65 \\
7.0 & 8-Aug-1991 & 8476 & 15-Aug-1991 & 8483 & $11.2^{\circ}$ & 2.72 \\
203.0 & 1-Dec-1992 & 8957 & 8-Dec-1992 & 8964 & $7.0^{\circ}$ & 1.75 \\
203.3 & 8-Dec-1992 & 8964 & 15-Dec-1992 & 8971 & $7.0^{\circ}$ & 1.75 \\
203.6 & 15-Dec-1992 & 8971 & 22-Dec-1992 & 8978 & $7.0^{\circ}$ & 1.69 \\
212.0 & 9-Mar-1993 & 9055 & 23-Mar-1993 & 9069 & $15.4^{\circ}$ & 2.71 \\
318.1 & 1-Feb-1994 & 9384 & 8-Feb-1994 & 9391 & $4.5^{\circ}$ & 1.78 \\
328.0 & 24-May-1994 & 9496 & 31-May-1994 & 9503 & $7.0^{\circ}$ & 1.56 \\
331.0 & 7-Jun-1994 & 9510 & 10-Jun-1994 & 9513 & $7.0^{\circ}$ & 0.95 \\
331.5 & 14-Jun-1994 & 9517 & 18-Jun-1994 & 9521 & $7.0^{\circ}$ & 1.34 \\
333.0 & 5-Jul-1994 & 9538 & 12-Jul-1994 & 9545 & $7.0^{\circ}$ & 1.86 \\
\hline
\end{tabular}


interval was performed independently using a variety of spatial distributions. These included models for the expected distribution of the galactic diffuse emission (based on the known gas distributions) and also empirical modeling using a superposition of one or more sources. Models for PSR 1951+32 (located $2.6^{\circ}$ away from Cyg X-1) were also included in the analysis. This pulsar has been detected by EGRET and there is evidence (based on a joint timing and spatial analysis) for it in the COMPTEL data as well [10]. Variations in the derived flux using different spatial models provided a handle on the systematic uncertainties in the analysis.

The COMPTEL spectrum for Cyg X-1 shows clear evidence for emission extending out to at least $2 \mathrm{MeV}$. These data alone can be modeled as a power law spectrum with a photon index of -3.7. Good fits can also be obtained using Comptonization models (with electron temperatures in the range of 450-700 $\mathrm{keV}$ ), but the extrapolation of these fits to lower energies is quite poor. Such values for the electron temperature are much higher than those derived from fits at lower energies. The COMPTEL data alone, therefore, suggest some inadequacy in the ability of the Comptonization models to fit the broad-band spectrum.

The COMPTEL spectrum (accumulated from the viewing periods listed in Table 1) is shown along with contemporaneous BATSE-EBOP and OSSE data in Figure 1. (BATSE-EBOP refers to an analysis of BATSE data using the JPL Enahnced BATSE Occultation Package [11].) This comparison demonstrates an inherent difficulty in precisely determining the shape of the spectrum near $1 \mathrm{MeV}$. In particular, the BATSE-EBOP data shows a clear trend towards higher flux levels, while the OSSE spectrum shows a clear trend toward lower flux levels. We are presently investigating the possibility that both trends (especially near $1 \mathrm{MeV}$ ) may be due to the presence of additional sources of emission in the Cygnus region. The COMPTEL analysis, for example, requires spatial modeling of several features in order to obtain a reliable spectrum. The emission in the region can be modeled either as a collection of (two or three) point sources or as a distribution which follows the general gas distribution within the galaxy. In either case, such emissions may have an impact on the spectra derived from both BATSE-EBOP and OSSE data. For example, the BATSE-EBOP spectrum is derived from an analysis which involves modeling various BATSE background components along with a large number of point sources distributed throughout the sky [11]. No provision is made for any diffuse emissions, which may be far more important at $1 \mathrm{MeV}$ than at $100 \mathrm{keV}$. Any galactic diffuse emission, if present, would therefore be included in the derived spectrum for Cyg X-1. Under the hypothesis of spatially distributed emission in the Cygnus region, the BATSE-EBOP spectrum would therefore tend to show unrealistically high flux levels. Likewise, the OSSE background subtraction process (on-source / off-source) would result in a background level that would be too high, leading to a reduced flux level in the derived Cyg X-1 spectrum. This type of trend is precisely what we 
observe. Whether or not it can explain the observed trends quantitatively (particularly near $1 \mathrm{MeV}$ ) is currently being investigated. The importance of this result is that observations with COMPTEL can be used to map out the spatial structure of emission near $1 \mathrm{MeV}$ and to more precisely pin down the $1 \mathrm{MeV}$ flux level. It may be that accurate spectral measurements near 1 $\mathrm{MeV}$ will require more detailed knowledge of the spatial distribution of the emission, information which only COMPTEL can easily provide.

\section{DISCUSSION}

Given the spectra presented in Figure 1, it is difficult to draw any firm conclusions about the nature of the spectrum near $1 \mathrm{MeV}$. As noted above, the COMPTEL data alone seems to further corraborate the conclusion that

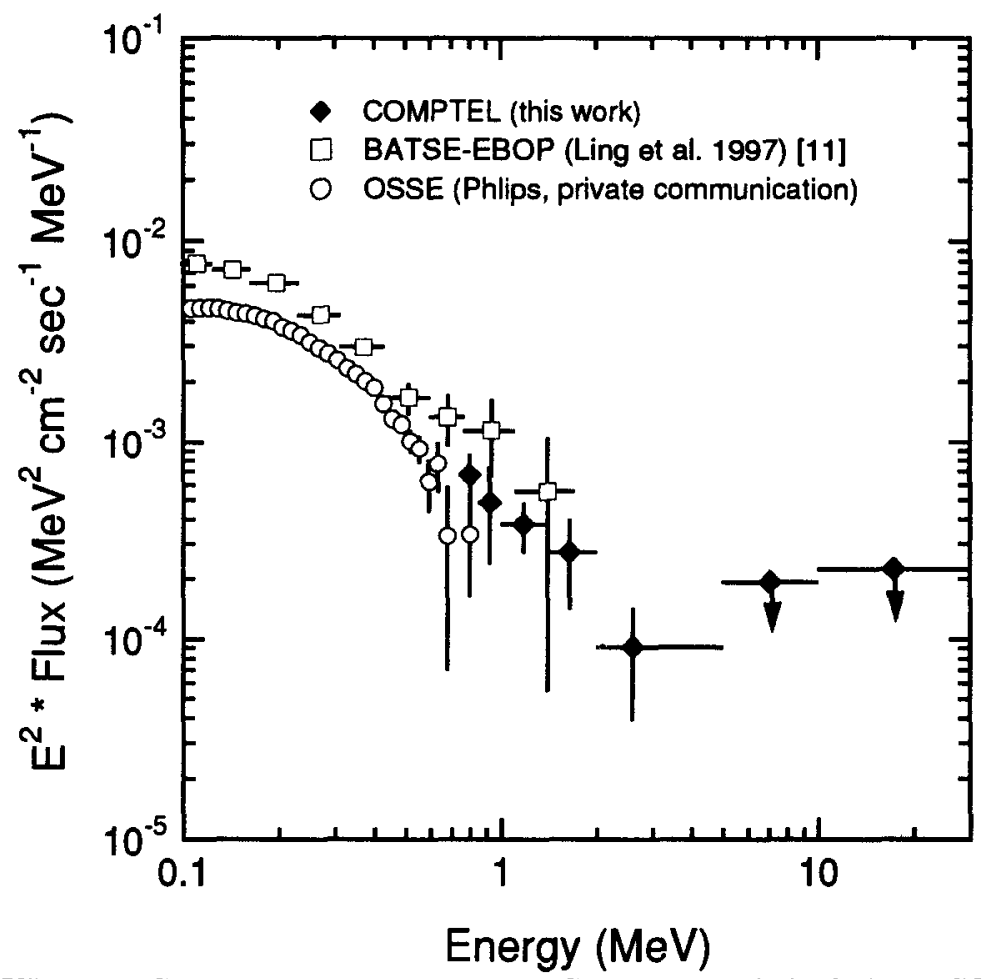

FIGURE 1. Contemporaneous spectra of Cyg X-1 as derived from COMPTEL, BATSE-EBOP and OSSE. Some OSSE upper limits have been removed for the sake of clarity. Error bars are $1 \sigma$. Upper limits are $2 \sigma$. Errors on COMPTEL data include estimates of systematic uncertainties due to spatial modeling of the COMPTEL images. 
standard thermal Comptonization models may be inadequate in describing the observed spectrum - Comptonization models fall off far too rapidly near 1 $\mathrm{MeV}$. A comparsion with BATSE-EBOP and OSSE data is difficult to make, given the discrepancies between the OSSE and BATSE-EBOP spectra. Independent fits to the BATSE/COMPTEL and the OSSE/COMPTEL spectra both lead to two conclusions: 1) the standard Comptonization models [1,2], when fit over the full range of the data, both fall off too rapidly to account for the flux near $1 \mathrm{MeV}$; and 2) improved fits with the Comptonization models can be obtained by limiting the fit to energies above $300 \mathrm{keV}$. These conclusions are both broadly consistent with those models which introduce Compton reflection. The reflection component in these models contributes only to energies below $\sim 300 \mathrm{keV}$, so that the fits we obtain at energies above $300 \mathrm{keV}$ may be a more realistic estimate of the electron temperature. On the other hand, stochastic acccleration models [9] predict a hard tail feature near $1 \mathrm{MeV}$ which may also be capable of modeling the COMPTEL results. Unfortunately, more detailed conclusions must await a resolution of the discrepancies between all of these data at energies below $1 \mathrm{MeV}$.

\section{ACKNOWLEDGEMENTS}

The COMPTEL project is supported by NASA under contract NAS5-26645, by the Deutsche Agentur für Raumfahrtgelenheiten (DARA) under grant 50 QV90968 and by the Netherlands Organization for Scientific Research NWO. We would also like to acknowledge both B. Phlips for providing the contemporaneous OSSE spectrum and J. Ling for providing the contemporaneous BATSE-EBOP spectrum.

\section{REFERENCES}

1. Sunyaev, R. A. \& Titarchuk, L. G. 1980, A\&A, 86, 121.

2. Titarchuk, L. 1994, ApJ, 434, 570.

3. Hua, X.M. \& Titarchuk, L. 1995, ApJ, 449, 188.

4. Skibo, J.G., et al. 1995, ApJ, 446, 86.

5. Haardt, F., Done, C., Matt, G., \& Fabian, A. C. 1993, ApJ Letters, 411, L95.

6. Wilms, J., Dove, J.B., Maisack, M., and Staubert, R. 1996, A\&A Supp., 120, C159.

7. Skibo, J.G. \& Dermer, C.D. 1995, ApJ Letters, 455, L25.

8. Ling, J.C., et al., 1997, ApJ, in press.

9. Li, H., Kusunose, M., \& Liang, E.P. 1996, ApJ Letters, 460, L29.

10. Hermsen, W., et al. 1997, Proc. 2nd INTEGRAL Workshop "The Transparent Universe", ESA SP-382, p. 287.

11. Ling, J.C., et al., 1997, ApJ Supp, submitted. 